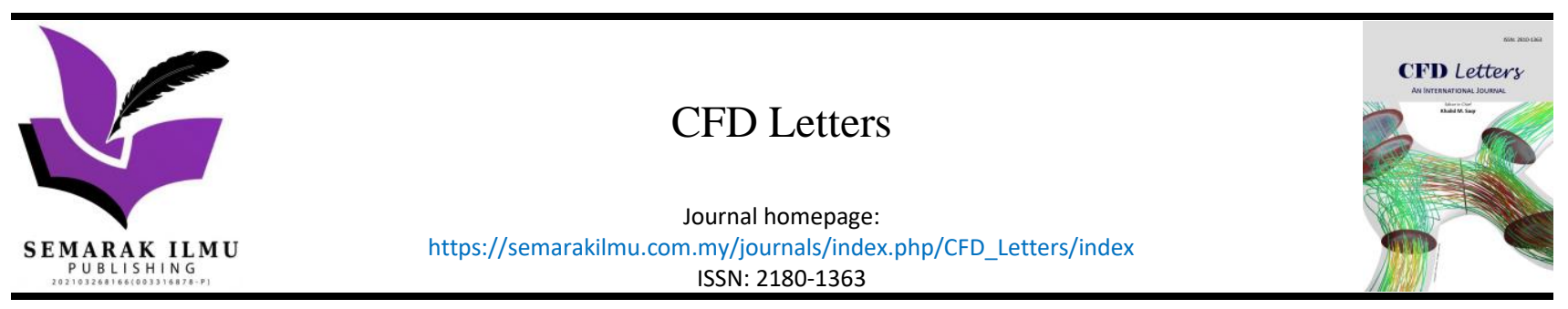

\title{
A CFD Analysis of Decommissioned Oil Platforms Jackets on the Brazilian Coast
}

\author{
Fernando Rodrigues Gonzalez ${ }^{1,}{ }^{*}$, Roger Matsumoto Moreira ${ }^{1}$ \\ Engineering School, Industrial Design Laboratory, Fluminense Federal University, Rua Passo da Pátria, 156, D block, room 563A, Niterói, RJ, CEP \\ 24210-240, Brazil
}

\section{ARTICLE INFO}

\section{Article history:}

Received 18 August 2021

Received in revised form 6 November 2021

Accepted 7 November 2021

Available online 14 December 2021

\section{Keywords:}

Decommissioning; artificial reefs; computational fluid dynamics (CFD); oil rigs

\section{ABSTRACT}

Every facility reaches the last phase in its life cycle, which is decommissioning. Since the last decade, this subject has been gaining importance in Brazil's offshore oil and gas companies. For jacket type rigs, one of the methods widely applied after idling the production is the conversion of these structures into artificial reefs (ARs). There are several critical aspects for choosing the best strategy for cutting and sinking a platform jacket, ensuring the success of an AR from a biological point of view. One of them is the influence of marine currents and their fluid-structure interaction which, by maximizing local upwelling and back vortex effects, favours the growth of aggregated flora and fauna. This study consists in the application of computational fluid dynamics (CFD) techniques for studying the marine flow around a disassembled and sunk jacket in the seabed for the purpose of converting it into an artificial reef. An FVM (Finite Volume Method) from a commercial software (most recent version of ANSYS FLUENT ${ }^{\circledR}$ ) is applied with the upwind scheme and a $k-\varepsilon$ turbulence model on steady state is chosen. Field data about Brazilian coastal currents are collected and analysed from the amount of information available on a Brazilian Navy's meteoceanographic program. Next, different combinations of cutting and sinking a jacket are studied, always keeping a minimum $55 \mathrm{~m}$ free water column. The objective is to verify where the formation of local upwelling regions - that is, where the vertical velocity component reaches values equal or greater than $10 \%$ of the magnitude of the free flow velocity - is more significant, without decreasing back eddy formation. It is observed that the dismemberment of the jacket with the positioning of its parts in an increasing height sequence in the direction of the prevailing current is favourable to generate local upwelling while tipping the structure at $90^{\circ}$ to the prevailing current results in the most voluminous back eddy region.

\section{Introduction}

\subsection{Purpose and Motivation}

Every production asset invariably reaches the last stage of its life cycle, which is the decommissioning of its facilities. In the current context, it is no longer considered acceptable to simply abandon the structures and equipment in the location without any type of study on the socio-

\footnotetext{
* Corresponding author.

E-mail adresse: fgonzalez@id.uff.br (Fernando Rodrigues Gonzalez)
} 
environmental impacts related to the emission of pollutants, soil, groundwater, rivers and seas degradation, in addition to the reduction of fauna and flora. For installations located far from land, very common in the oil and gas sector, one of the main technological challenges (and cost aggregators) in decommissioning is the deployment of large structures (such as jackets, topsides, pipes and subsea equipment) on land, where the most likely destination will be its dismantling and recycling of scrap material. To reduce the complexity of decommissioning projects, there are some alternatives for reusing offshore structures, such as assembling wind turbines for power generation, conversion to military bases, scientific research stations and even hotels for recreational fishing.

As pointed out by several authors, such as Kaiser, [1] and Zalmon et al., [2], one of the alternatives that has been shown to be quite advantageous is the disassembly and sinking of these structures aiming the formation of artificial reefs (ARs), which greatly reduces the cost of maritime transport while favoring the enrichment of the marine biology. Socioeconomic gains are also important, considering the benefits to fishing coastal communities, in addition to encouraging ecotourism and sport fishing. Several experiences were conducted with the creation of ARs around the world, using from inoperative ships [3] to used tires, concrete blocks [2] and structures designed specifically for this purpose. Figure 1 shows an example of a reefed jacket in the context of the Rig-to-Reefs program on the Gulf of Mexico (USA) [4].

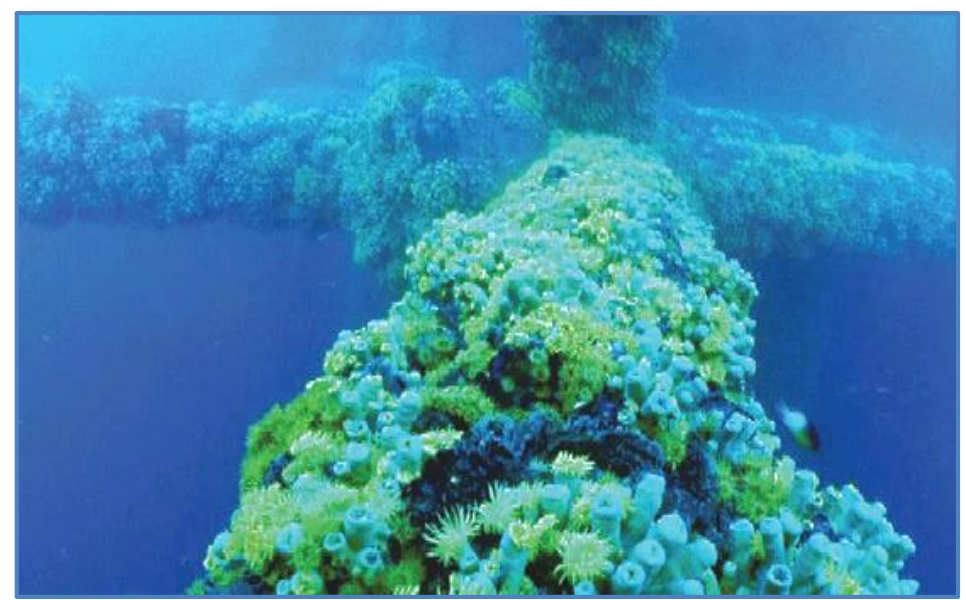

Fig. 1. Reefed jacket on the Gulf of Mexico (USA) [4]

In Brazil, the issue of offshore rigs decommissioning has been gaining importance since the last few years. Numerous fixed-type rigs are maintained in operation, generally supported by framework structures called jackets. Several of these units started operating more than 30 years ago, and many of them are likely to be decommissioned in the coming years.

Several aspects shall be considered for the success of an AR project, for example, materials, structural integrity, temperature, turbidity, salinity of the water, presence of nutrients, socioeconomic and cost impacts [3]. One of these important subjects to be regarded is the fluidstructure interaction and the resultant flow behavior.

Back in 1989, Baynes \& Szmant, [5] developed field research determining the effects of sea currents on the benthic communities of an AR located off the coast of Florida (planned shipwreck of the Biscayne boat at a depth of $20 \mathrm{~m}$ ). The authors concluded that biodiversity is a function of the marine current magnitude, where higher velocities promote a renewal of nutrients and avoid sedimentation, which is unfavorable to the settlement and colonization of new organisms. However, there is a limit, around $50 \mathrm{~cm} / \mathrm{s}$, for which the larvae become unable to attach themselves to the substrate. It is also recommended to try to maximize the laminar flow, as it was observed a reduction in biomass in places with excessive recirculation, due to the tendency to accumulate sediments. 
Several authors, such as Lindberg, Seaman \& Zimmerman [6], Ito [7], and Zheng et al., [8], discoursed about the benefic effects of localized upwelling flow generation. Liu et al., [9] defined the upwelling region where the relation $v / U_{\infty}$ is bigger than 0.1 , that is, the vertical velocity $(v)$ reaches values of, at least, $10 \%$ of the distant marine current velocity $\left(U_{\infty}\right)$. The authors also determined the optimal ratio between the height of the AR and the total depth in a way to maximize local upwelling, obtaining a value of 0.2 for this relationship. Later, Liu et al., [10] performed several numerical simulations using star-shaped models placed alone or combined in arrangements of two equal bodies, testing the effects of increasing the distance between them as well as the arrangement of the pairs on the sea floor. Zheng et al., [8], proposed a very similar analysis, but this time using a three tubes geometry in side-by-side arrangements. They also conducted experimental bench tests to confirm the results, obtaining a mean deviation smaller than $10 \%$ in numerical simulations for the following characteristics: upwelling region height $\left(H_{U P}\right)$, upwelling region area ( $\left.S_{U P}\right)$, maximum upwelling velocity $\left(v_{\max }\right)$ and back eddy region area $\left(S_{\text {back }}\right)$.

Nevertheless, it was only in 2021 that a geometric model of a platform jacket converted to a RA was used in CFD studies conducted by Rahman et al., [11, 12]. In these works, the efficiency of the sinking method (that is, the direction in which the jacket is tipped) in terms of upwelling and back vortex generation are explored, as well as other parameters, such as drag, lift forces and pressure distribution.

Although the phenomenon called upwelling is treated, in the oceanography literature, as a macroecological phenomenon generated by the dynamics of cold and hot currents, or also caused by the actions of the wind (formation of Ekman spirals) among other phenomena [13], the effect of local upsurges around artificial reefs is also relevant, as revealed by the above-mentioned authors. Thus, the concept of the term upwelling applied in this study is used in the context of the localized effects caused by the fluid-structure interaction.

Regarding back eddy generation, Baynes \& Szmant [5] alerted for a sedimentation tendency in regions with excessive recirculation, thus observing less organisms in these areas. However, several authors, such as Kim et al., [14], endorses the importance of wake regions for fish species, that are attracted by shelter, feeding and spawning grounds, rest areas, or temporary stopovers.

Other lines of study on the hydrodynamics around ARs were followed, as in the paper by Jiang et al., [15], who conducted experimental bench tests for hollow cubic shapes with circular holes and drew comparisons between the values of forces and speeds obtained numerically. Yaakob et al., [16] simulated different AR formats considering Malaysian sea conditions and compared the traditional hollow cube shape with a model similar to a cyclist's helmet, choosing the latter as more efficient due to its low drag coefficient and, therefore, less tendency to form stagnation and high-pressure zones.

Just recently, some few authors have made CFD studies using a geometry originated from a fixedplatform jacket converted to an RA. Also, it wasn't found any investigation in the literature about side-by-side arrangements of different structures or jacket sections. It's expected that the results obtained may help the industry when carrying out decommissioning endeavors, reducing some of its uncertainties, observing the particularities of each case. The possible expansion of the number of platforms eligible for conversion into RAs, as well as the possibility of making them more efficient in the production of biodiversity, also potentializes benefits for a greater number of coastal communities whose economic activity is closely linked to fishing, also bringing benefits for aquatic ecotourism and practice of sport fishing.

The purpose of this paper is to analyze the hydrodynamic profile generated by the fluid-structure interaction in a hypothetical jacket-type platform converted to an artificial reef using CFD techniques. Different alternatives for decommissioning and sinking are outlined, always aiming to increase the 
efficiency of the AR in accordance with the physical and biological principles already explored by the scientific community. After a brief bibliographic review, a general description of the problem and boundary conditions are explained, succeeded by a mathematical modeling for the numerical solution. To choose the best turbulence model in steady state, numerical simulations are performed with a geometry adapted from the literature, seeking to obtain the less deviations in comparison with experimental data provided by its authors.

\section{Methodology}

\subsection{Problem Overview}

The physical problem addressed consists of a jacket structure typically used for oil production in shallow water, disassembled and sunk for conversion to an AR. Thirteen configurations are studied, being the first five characterized by simply toppling the jacket after cutting its legs close to the sea bottom, varying the angle $\theta$ of incidence to the prevailing current (see Figure 2). The next four ways are characterized by disassembling it in two parts, and positioning side by side aligned with the predominant flow at separation distances of $0.5 L_{b}, 1.0 L_{b}, 1.5 L_{b}$ and $2.0 L_{b}$, where $L_{b}$ is the width of the jacket base, in similarity to the job done by Liu et al., [10] (see Figure 3). The last four ways represents cutting the jacket in three parts also spaced in the same manner (see Figure 4).

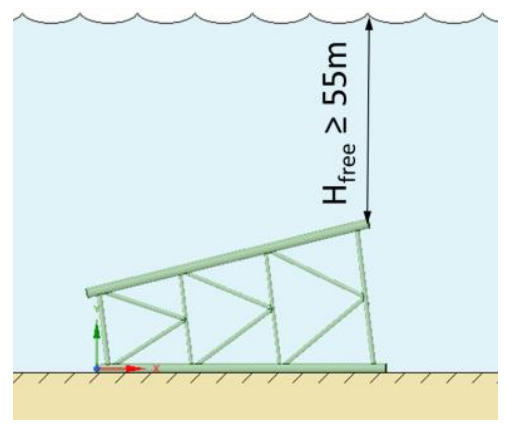

(a)

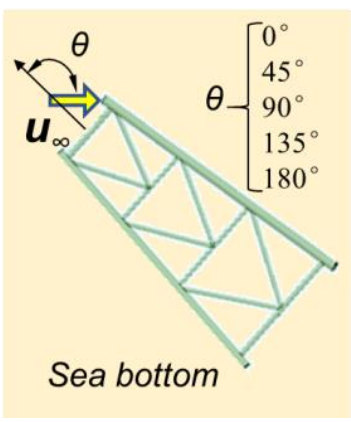

(b)

Fig. 2. Schematic representation of the toppled platform on the seabed: (a) side view (b) upper view

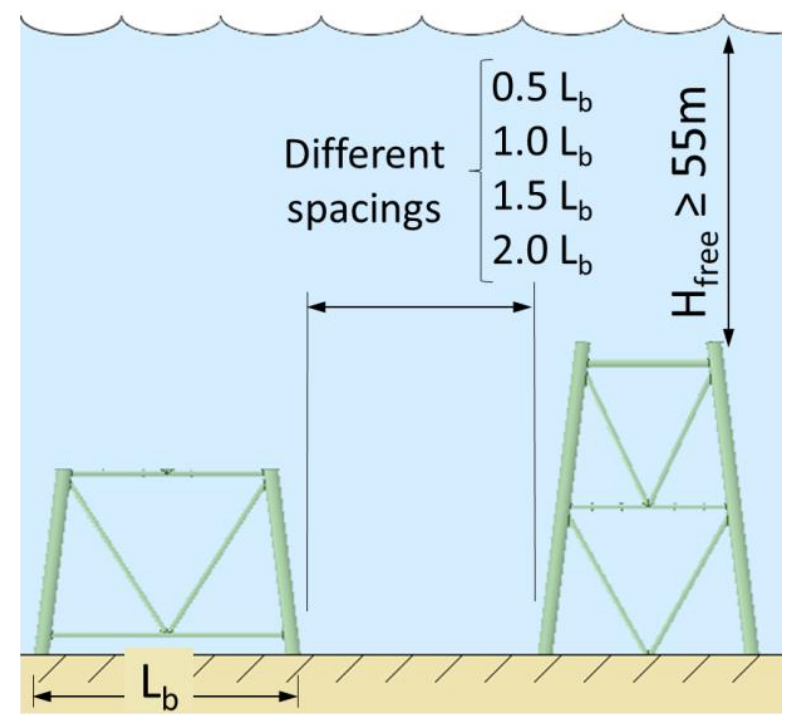

Fig. 3. Schematic representation of the platform cut in two parts 


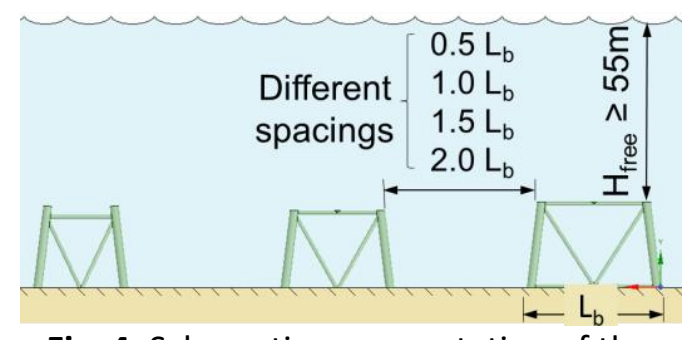

Fig. 4. Schematic representation of the platform cut in three parts

Considering the lack of a specifical policy for Artificial Reefs in Brazil, it will be respected, for all cases, the limit imposed by the item 3.6 of Regulation A.672 (16) of the IMO (International Maritime Organization [17], which recommends maintaining a free water column of at least 55 meters, thus avoiding impact on local navigability. Even though Brazilian ports authorities limit the draught of marine vessels to $20 \mathrm{~m}$ (case of Port of Itaguaí-RJ), that question remains unclear when it comes to license an AR project

The objective of this study is to evaluate and compare the formation of local upwelling and back eddy regions formed around these structures, seeking to maximize both. Upwelling regions are considered where $v>0.1 U_{\infty}$ and eddy regions are considered where $u<0$.

\subsection{Boundary Value Problem}

To simulate representative conditions of the sea flow, a parallelepiped domain is built as shown in Figure 5.

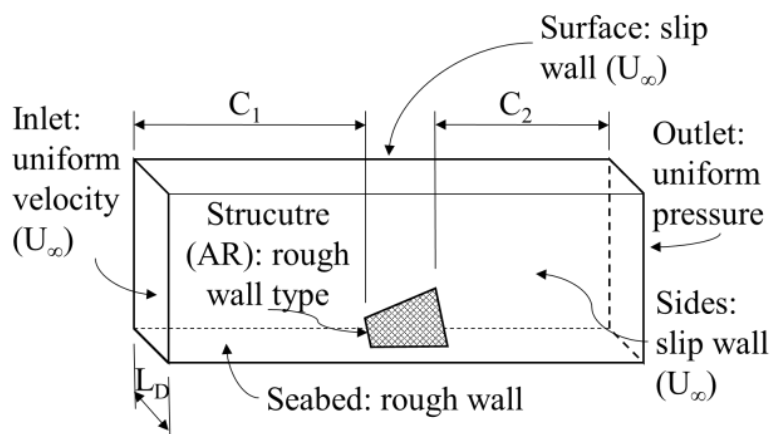

Fig. 5. Boundary conditions

Gravitational effects are neglected to simplify the analysis, as it is a predominantly horizontal flow without buoyancy and wave effects. The domain is also considered to be of constant depth, since the objective is only to verify the interaction of the flow with the jacket structure, without worrying about possible variations in the undersea topology. Another simplification imposed is the application of a steady condition, understanding it as being sufficient for a mere comparative analysis.

As shown in Figure 5 , the distances $C_{1}, C_{2}$ and $L_{D}$ must guarantee fully developed flow conditions as a simplified approach to the marine environment, in addition to avoiding the interference of the side walls in the analysis. Considering the model used by Zheng et al., [8], and the small deviations to the experimental data, a domain with proportional dimensions is constructed, that is: $C_{1}=5 C, C_{2}=$ $10 C$ and $L_{D}=10 C$, where $C$ is the jacket height, as can be seen in Figure 6 . 


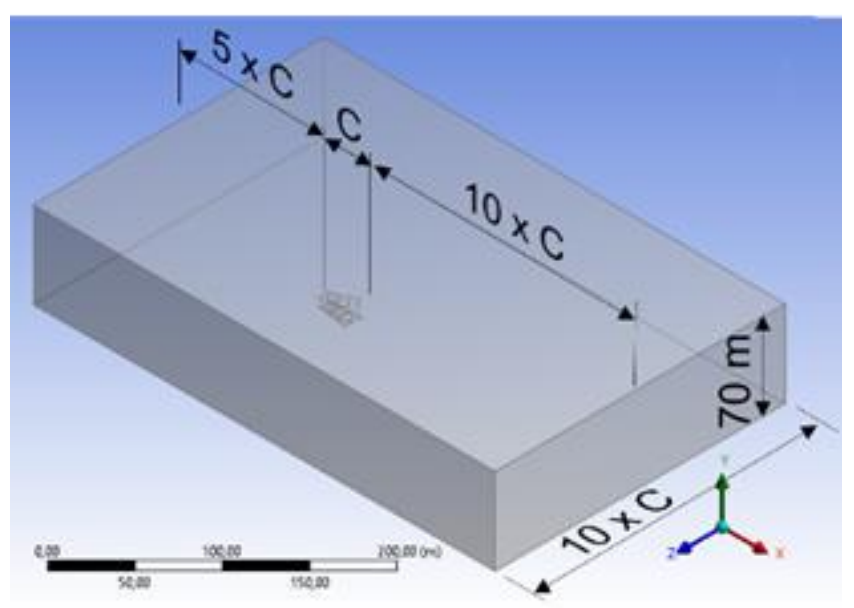

Fig. 6. Domain dimensions

The governing equations (in Einstein notation) to be considered for an incompressible Newtonian fluid are

$\partial u_{i} / \partial x_{i}=0$

$\frac{\partial u_{j} u_{i}}{\partial x_{j}}=f_{i}-\frac{1}{\rho} \frac{\partial P}{\partial x_{i}}+v \frac{\partial^{2} u_{i}}{\partial x_{j}^{2}}$

where $v$ is the kinematic viscosity and $f$ is the source term, represented by the vector $(0,0,-g)$, which is irrelevant in this case.

In the turbulent model, the momentum conservation equations can be represented by the Reynolds Averaged of the Navier-Stokes Equations (RANS), where the velocity and pressure fields are calculated as their mean values plus the fluctuating components as below

$u_{i}\left(x_{i}, t\right)=\bar{u}_{i}\left(x_{i}\right)+u_{i}^{\prime}\left(x_{i}, t\right)$

$P\left(x_{i}, t\right)=\bar{P}\left(x_{i}\right)+P^{\prime}\left(x_{i}, t\right)$

To solve the RANS equations, it is necessary to apply specific turbulence models. The more commonly used in CFD packages are $k-\varepsilon$ (standard and RNG - Renormalization Group); $k-\omega$ and SST (Shear Stress Transport). Further ahead, numerical results will be compared with the experimental data found in literature, showing that the $k-\varepsilon$ model has the smallest deviations for a steady flow simulation. The two main equations in the standard $k-\varepsilon$ model are

$\partial(\rho k){ }_{\partial t}+\boldsymbol{\nabla} \cdot(\rho k \overline{\boldsymbol{u}})=\boldsymbol{\nabla} \cdot\left[\left(\mu+\mu_{T} / \sigma_{k}\right) \nabla k\right]+2 \mu_{T} \boldsymbol{E}_{\boldsymbol{i j}} \cdot \boldsymbol{E}_{\boldsymbol{i j}}-\rho \varepsilon$

$\partial(\rho \varepsilon) / \partial t+\nabla \cdot(\rho \varepsilon \overline{\boldsymbol{u}})=\boldsymbol{\nabla} \cdot\left[\left(\mu+\frac{\mu_{T}}{\sigma_{\varepsilon}}\right) \nabla \varepsilon\right]+C_{1 \varepsilon} \frac{\varepsilon}{k} 2 \mu_{T} \boldsymbol{E}_{\boldsymbol{i j}} \cdot \boldsymbol{E}_{\boldsymbol{i j}}-\rho C_{2 \varepsilon} \frac{\varepsilon^{2}}{k}$

where $E i j$ is the stress tensor: $\frac{1}{2}\left(\frac{\partial u_{i}}{\partial x_{j}}+\frac{\partial u_{j}}{\partial x_{i}}\right)$. 
These two equations are closed by the relationship between turbulent dynamic viscosity and the variables $k$ and $\varepsilon$

$\mu_{T}=\rho C_{\mu} k^{2} / \varepsilon$

The constants in the standard $k-\varepsilon$ model are experimental values found in literature: $\sigma_{k}=1.0, \sigma_{\varepsilon}=$ $1.3, C_{\mu}=0.09, C_{\varepsilon 1}=1.44$ and $C_{\varepsilon 2}=1.92$.

\subsection{Finite Volume Method (FVM)}

The FVM consists in the division of the domain in a finite number of control volumes for the application of the conservation equations in each one. This technique allows converting vector and continuous transport equations into discrete ones that can be solved numerically in an iterative way. Several discretized equations can be assembled for each cell, thus forming a matrix that can be solved by the computational algorithm. It is used the Gauss-Seidel method in conjunction with an algebraic multigrid method for solving the system of equations.

To establish the relationship between the value of one scalar quantity $\varphi$ at the center of one cell and at the center of its adjacent cell, it is necessary to determine its value on the interface $\left(\varphi_{f}\right)$ for the solution of the convective terms. This value can be calculated by several methods, with the first order upwind scheme being more commonly used. In that method, $\varphi_{f}$ assumes the value of $\varphi$ in the center of the upstream cell, regarding the flow direction. If the pressure field were known, it would be enough to calculate the velocity field. However, in most fluid dynamic problems, the pressure distribution and mass flows on the faces are unknown. The discretized equation requires the face pressure value between the cells, thus requiring an interpolation scheme to calculate the face pressure values from the cell values. The most popular numerical solution algorithms for coupling the velocity and pressure fields are the SIMPLE (Semi-Implicit Method for Linked Equations) and SIMPLEC (Semi-Implicit Method for Linked Equations - Consistent) schemes [18].

\subsection{Field Data Analysis}

To determine the subsea flow velocity, a study is made on Brazilian currents in different regions using information from the meteoceanographic buoys (PNBOIA program) available at the Brazilian Navy website [19]. After processing the information, including the exclusion of spurious data according to the quality criteria established by the Navy itself, the predominant velocities on Brazilian coast obtained vary from 0.28 to $0.64 \mathrm{~m} / \mathrm{s}$ at depths ranging from 10.5 to 13.0 meters. Considering the Reynolds number for this velocity range $\left(3.3 \times 10^{6}\right.$ to $\left.7.5 \times 10^{6}\right)$, the turbulent flow can be considered as fully developed [18]. Thus, since it is a comparative analysis, it is not necessary to carry out simulations on different magnitudes of velocities and an average value for the current velocity $(0.45 \mathrm{~m} / \mathrm{s})$ is adopted.

Using the same meteoceanographic database, it is possible to extract the peak periods $\left(T_{P}\right)$ of the sea waves in each of the analyzed regions. For the data considered as valid, the value of the wavelength $(L)$ is calculated, according to Sorensen [20]

$$
L=g T_{P}^{2} / 2 \pi
$$


Eq. (8) is applied and the following values for half wavelength ( $L / 2)$ are obtained, as shown in Table 1

\section{Table 1}

Most frequent wavelengths found in the Brazilian coast

\begin{tabular}{lll}
\hline Buoy Location & Most frequent Wave Periods $\left(T_{p}\right)$ range $(\mathrm{s})$ & Average $L / 2(\mathrm{~m})$ \\
\hline Fortaleza & 6.2 to 7.2 & 35 \\
Recife & 7.2 to 8.0 & 45 \\
P. Seguro & 6.2 to 7.2 & 55 \\
Vitória & 6.2 to 8.0 & 40 \\
Itaoca & 6.2 to 7.2 & 35 \\
Cabo Frio & 6.2 to 7.2 & 35 \\
Santos & 7.2 to 8.0 & 45 \\
\hline
\end{tabular}

By observing the available data, one can conclude that the most frequent $L / 2$ parameter reaches $55 \mathrm{~m}$ in some cases, but for most part it does not exceed $35 \mathrm{~m}$. Sorensen [20] defines that, for depths greater than $L / 2$, one can consider it as deep wave region, where the influence of surface waves is practically null.

\subsection{Comparison with Literature for Turbulence Model Selection}

To select the best turbulence model in steady state, a comparison is made with experimental data from literature. It is chosen the work of Liu et al., [9] due to 1) approximation of a jacket-type platform geometry, which is also a hollow shape and 2) it has the same subject of interest of the present work, that is, the formation of upwelling and back eddy regions. Figure 7 shows the geometric model constructed in similarity to the author's referred article.

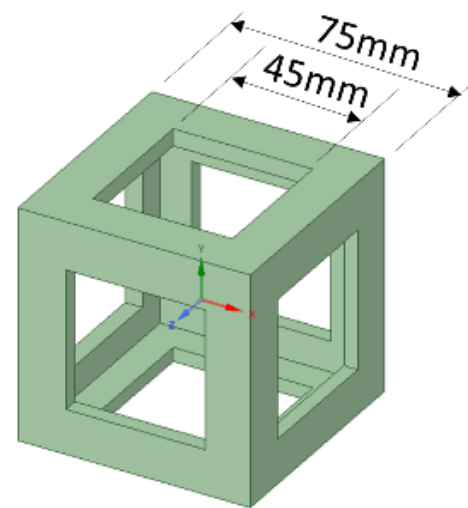

Fig. 7. Geometry developed in similarity to work by Liu et al., [9]

Different turbulence models are tested: RNG $k-\varepsilon$, standard $k-\varepsilon$, standard $k-\omega$ and SST, obtaining different results for $H_{u p} / H, S_{u p} / S, v_{a v g}$ (average vertical velocity) and $S_{\text {back }} / S$, as represented in Figure 8 below 


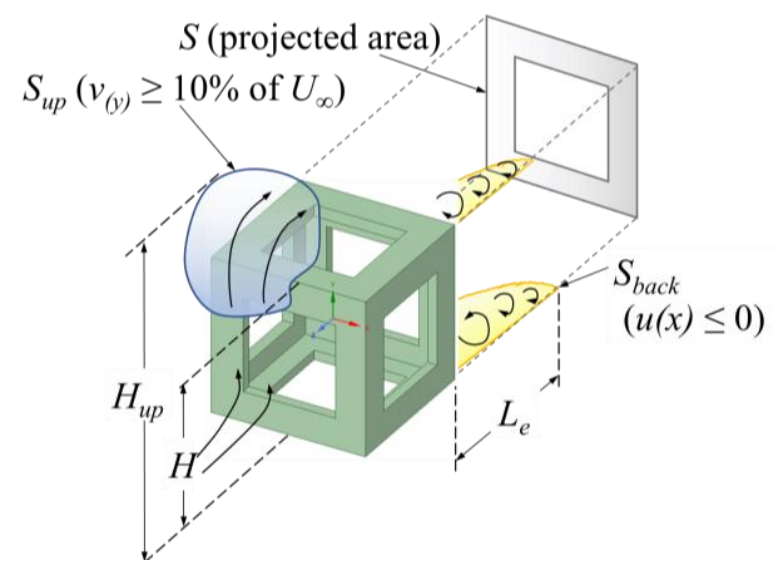

Fig. 8. Main flow characteristics on the AR model studied by Liu et al., [9]

The deviations to the experimental results are shown in Figure 9. By observing the results, the $k$ $\varepsilon$ model is chosen for the jacket simulation

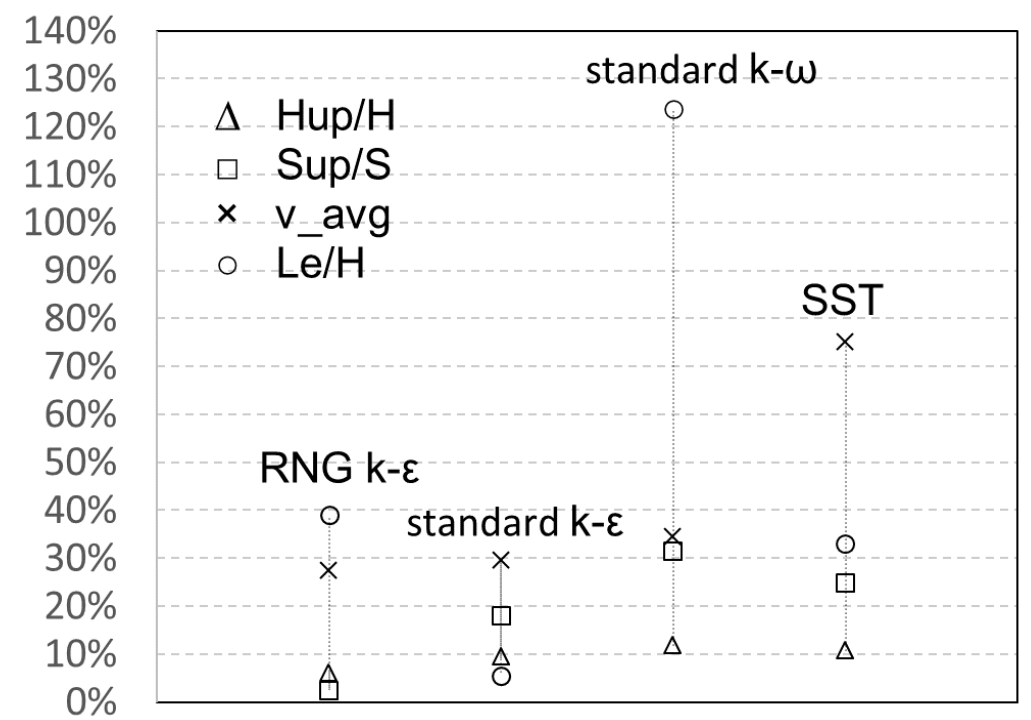

Fig. 9. Deviations obtained in comparison with the experiment LIU et al., [9]

\subsection{Turbulent Initial Parameters}

The following parameters are estimated for the domain inlet and outlet surface

i. Intensity of turbulence $\left(I_{c}\right)$ : as recommended by Veritas [21], in case of high roughness, a value of $6.5 \%$ is assumed as an initial value.

ii. Initial Viscosity Rate $\left(\mu_{T} / \mu\right)$ : it is applied an initial guess of 10 (dimensionless).

The initial values for $k$ and $\varepsilon$ can be estimated by the following equations

$k=3 / 2\left(U_{\infty} I_{C}\right)^{2}$ 
$\varepsilon=\rho C_{\mu} k^{2} / \mu_{T}$

Roughness for the seabed is hypothetically assumed to be equivalent to the medium sand grain size $(0.0004 \mathrm{~m})$, as indicated by Veritas [21]. For the jacket surface, it is assumed a roughness equivalent for minimum marine growth $(0.005 \mathrm{~m})$, according to the same reference. Roughness coefficient for a uniform distribution is adopted (for comparative reasons).

The jacket is positioned in the domain representing some penetration of the structure on the marine soil. As this depends on a series of factors such as composition of the marine soil, mass of the structure, among others, a penetration equivalent to half the diameter of the jacket leg pipe is adopted $(0.425 \mathrm{~m})$.

\subsection{Mesh Construction and Grid Independence Test}

It is used a non-structured mesh with near wall treatment using inflation layer, in a way to capture boundary layer effects of the sea bottom, similarly to the strategy adopted by Ali et al., [22]. According to Pope [23], the boundary layer in turbulent regime has several sublayers in which the domain of the viscosity effect is reduced according to the distance $(\Delta y)$ from the wall. It is then possible to calculate the distance $\Delta y$ from the centroid of the mesh element to the wall region, according to the equation below

$\Delta y=y^{+} v / u_{\tau}$

where $y^{+}$is the dimensionless distance from the wall and $u_{\tau}$ is the frictional velocity, given by

$u_{\tau}=\sqrt{\tau_{w} / \rho}$

where $\tau_{w}$ is the shear stress on the wall, which can be calculated as follows

$\tau_{w}=\frac{1}{2} C_{f} \rho U_{\infty}^{2}$

where $C_{f}$ is the friction coefficient, which is obtained by the following equation [24]

$C f=\left[1,89+1,62 \log \left(l / k_{s}\right)\right]^{-2,5}$

where $k_{s}$ is the equivalent roughness and $l$ is the length of the plate or the region covered by the fluid. According to Pope [23], for $y^{+}>50$, viscous effects become practically null. As the Reynolds number value is relatively high and there is no predominance of viscosity effects close to the wall, $y^{+}$values in a range of 500 to 1000 are adopted.

The size of the elements close to wall regions varies from $0.04 \mathrm{~m}$ to $0.08 \mathrm{~m}$ for the grid independence test. At distant regions, in which the flow is practically undisturbed, a particular element size is 100 times greater than other one near the wall region, with a gradual growth factor equal to 1.2. Figure 10 shows details of the constructed mesh. 


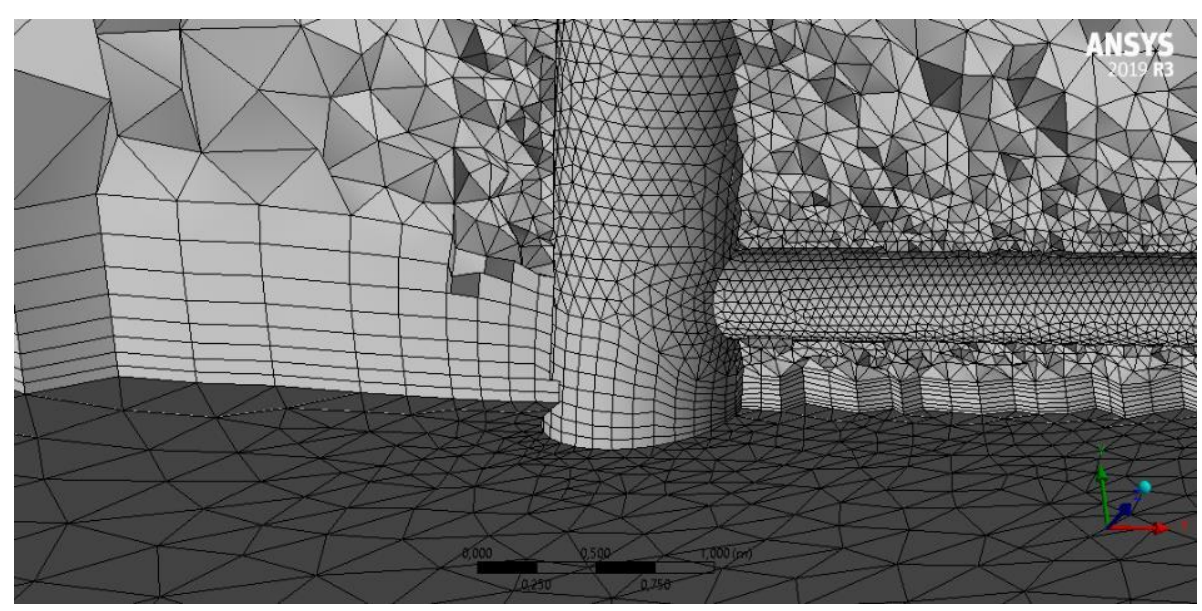

Fig. 10. Mesh detail with near wall inflation treatment

Aiming to establish an optimal number of mesh elements in a way to produce a stabilized result avoiding high computational costs, the same model (toppled jacket aligned to $180^{\circ}$ with the flow) is simulated several times by varying the number of nodes and, proportionally, the number of mesh elements from $4.5 \times 10^{6}$ to $12 \times 10^{6}$ elements. A steady state model is used, with 1000 iterations (for which a tendency to stabilize residual values is verified), using the SIMPLEC algorithm and other parameters adjusted as previously described. Two control points $\left(P_{1}\right.$ and $\left.P_{2}\right)$ are considered for the evaluation of velocity values. These points were chosen after watching areas where upwelling and recirculation effects coexists in preliminary simulations.

By observing the behavior of the velocity vector module and its components at these two points, one can conclude that there are significant variations when the mesh is coarser. However, the back eddy $(u<0)$ and local upwelling $\left(v>0.1 U_{\infty}\right)$ regions remain equally characterized for $P_{1}$ and $P_{2}$ in all mesh densities. Regarding the main characteristics studied, there is a significant variation in the upwelling and back eddy volumes below $9 \times 10^{6}$ elements. When the number of elements rises above that, the variations in those characteristics are irrelevant. However, the calculation time increases significantly ( $200 \%$ more). The residual values of the numerical iterations also tend to stabilize with $9 \times 10^{6}$ elements, allowing to conclude that little gain is obtained with excessive mesh refinement.

To maintain the number of mesh elements, close to $9 \times 10^{6}$, the following characteristics are adopted for all simulation conditions: 1 ) tetrahedral elements far from surfaces and inflation near sea bottom; 2) smaller mesh element size at curved surface regions $=0.05 \mathrm{~m}$; 3) larger size of the mesh element in the distant flow $=5 \mathrm{~m} ; 4$ ) growth factor of the mesh elements $=1.2$.

\section{Results}

\subsection{Simulation Results in Different Cutting and Sinking Conditions}

The thirteen ways of cutting and sinking the jacket are shown in Table 2 
Table 2

Different jacket cut and sink arrangements

\begin{tabular}{lll}
\hline Case & Cut and sink method & Angle with $U_{\infty}$ or distancing between parts \\
\hline 1.a & Base cut / toppling & $0^{\circ}$ \\
1.b & Base cut / toppling & $45^{\circ}$ \\
1.c & Base cut / toppling & $90^{\circ}$ \\
1.d & Base cut / toppling & $135^{\circ}$ \\
1.e & Base cut / toppling & $180^{\circ}$ \\
2.a & 2 parts side-by-side (cut at 40\% of height) & $0.5 L_{b}$ \\
2.b & 2 parts side-by-side (cut at 40\% of height) & $1.0 L_{b}$ \\
2.c & 2 parts side-by-side (cut at 40\% of height) & $1.5 L_{b}$ \\
2.d & 2 parts side-by-side (cut at 40\% of height) & $2.0 L_{b}$ \\
3.a & 3 parts side-by-side (two cuts) & $0.5 L_{b}$ \\
3.b & 3 parts side-by-side (two cuts) & $1.0 L_{b}$ \\
3.c & 3 parts side-by-side (two cuts) & $1.5 L_{b}$ \\
3.d & 3 parts side-by-side (two cuts) & $2.0 L_{b}$ \\
\hline
\end{tabular}

Using the conditions and assumptions previously defined, the results presented for relative height of the upwelling region $\left(H_{\text {up }} / H\right)$, relative volume of the local upwelling region $\left(V_{u p} / V\right)$ and relative volume of the back eddy region $\left(V_{\text {back }} / V\right)$ are shown in Tables 3, 4 and 5, below

Table 3

Results obtained for case 1 (toppling of the structure), $U_{\infty}=0.45 \mathrm{~m} / \mathrm{s}$

\begin{tabular}{llllll}
\hline Case & $1 . a$ & $1 . b$ & $1 . c$ & $1 . d$ & $1 . e$ \\
\hline Toppling angle & $0^{\circ}$ & $45^{\circ}$ & $90^{\circ}$ & $135^{\circ}$ & $180^{\circ}$ \\
\hline$H_{\text {up }} / H$ & 1.07 & 1.06 & 1.06 & 1.05 & 1.02 \\
$V_{\text {up }} / \mathrm{V}$ & 2.02 & 2.83 & 4.29 & 2.69 & 1.59 \\
$V_{\text {back }} / \mathrm{N}$ & 0.14 & 0.11 & 0.47 & 0.10 & 0.14 \\
\hline
\end{tabular}

Table 4

Results obtained for case 2 (jacket cut in 2 parts), $U_{\infty}=0.45 \mathrm{~m} / \mathrm{s}$

\begin{tabular}{lllll}
\hline Case & $2 . a$ & $2 . b$ & $2 . c$ & $2 . d$ \\
\hline Spacing $(\mathrm{m})$ & $0.5 L_{b}$ & $1.0 L_{b}$ & $1.5 L_{b}$ & $2.0 L_{b}$ \\
\hline$H_{\text {up }} / H$ & 1.07 & 1.07 & 1.07 & 1.07 \\
$V_{\text {up }} / V$ & 2.97 & 3.20 & 3.22 & 2.72 \\
$V_{\text {back }} / V$ & 0.23 & 0.18 & 0.23 & 0.24 \\
\hline
\end{tabular}

Table 5

Results obtained for case 3 (jacket cut in 3 parts), $U_{\infty}=0.45 \mathrm{~m} / \mathrm{s}$

\begin{tabular}{lllll}
\hline Case & $3 . a$ & $3 . b$ & $3 . c$ & $3 . d$ \\
\hline Spacing $(\mathrm{m})$ & $0.5 L_{b}$ & $1.0 L_{b}$ & $1.5 L_{b}$ & $2.0 L_{b}$ \\
\hline$H_{\text {up }} / H$ & 1.20 & 1.27 & 1.27 & 1.15 \\
$V_{\text {up }} / V$ & 6.18 & 5.53 & 4.80 & 2.40 \\
$V_{\text {back }} / V$ & 0.22 & 0.23 & 0.23 & 0.22 \\
\hline
\end{tabular}

It is observed that the localized upwelling regions are significantly voluminous and higher when cutting the structure in three parts and positioning them nearby (Figure 11). However, when increasing the separation between the parts of the jacket, it causes the flow characteristics to change significantly, reducing the upwelling isovolume. The lowest and smaller upwelling region is observed with the jacket toppled at $180^{\circ}$ in relation to the prevailing current (Figure 12).

The complete comparison of upwelling relative volume and height are shown in graphs given by Figures 13 and 14, respectively. 


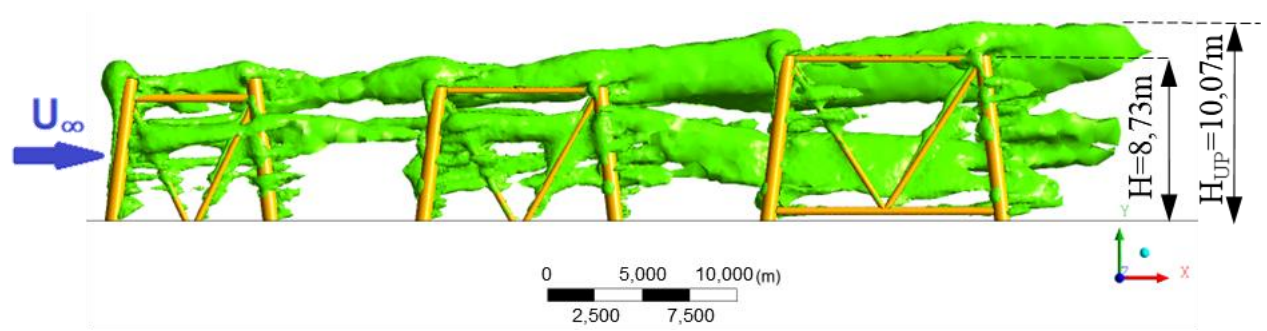

Fig. 11. Upwelling isovolume generated by the flow at $0.45 \mathrm{~m} / \mathrm{s}$ passing through the structure cut into 3 parts spaced by $0.5 L_{b}$

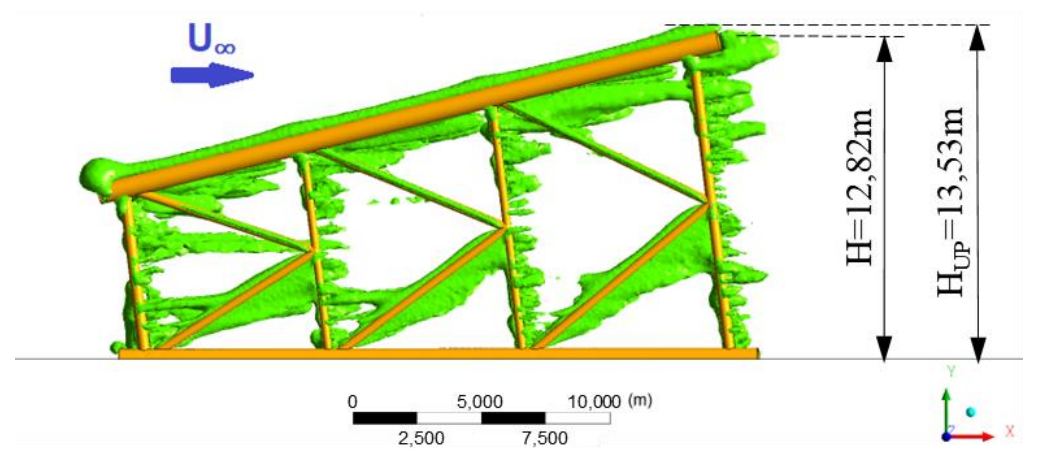

Fig. 12. Upwelling isovolume generated by the flow at $0.45 \mathrm{~m} / \mathrm{s}$ passing through the toppled jacket at $180^{\circ}$ to the prevailing current

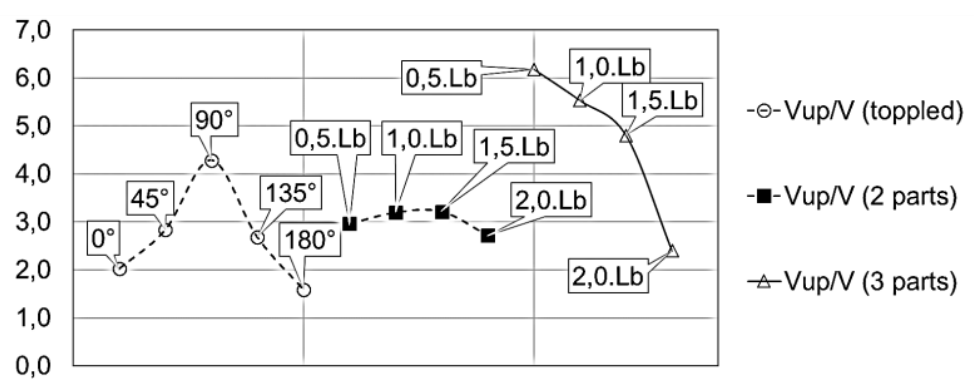

Fig. 13. Variation of $V_{u p} / V$ for each case

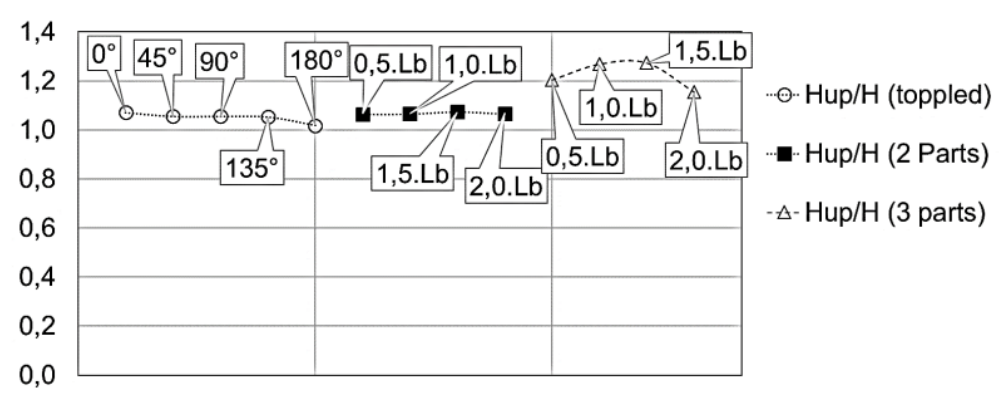

Fig. 14. Variation of $H_{u p} / H$ for each case

An alternative that also presents good results is the execution of the toppling in an angle of $90^{\circ}$ to the prevailing current. In this condition, the local upwelling region is more voluminous than toppling the jacket streamwise $\left(0^{\circ}\right.$ or $\left.180^{\circ}\right)$, despite being relatively low in relation to the height reached, as shown in Figure 15. 


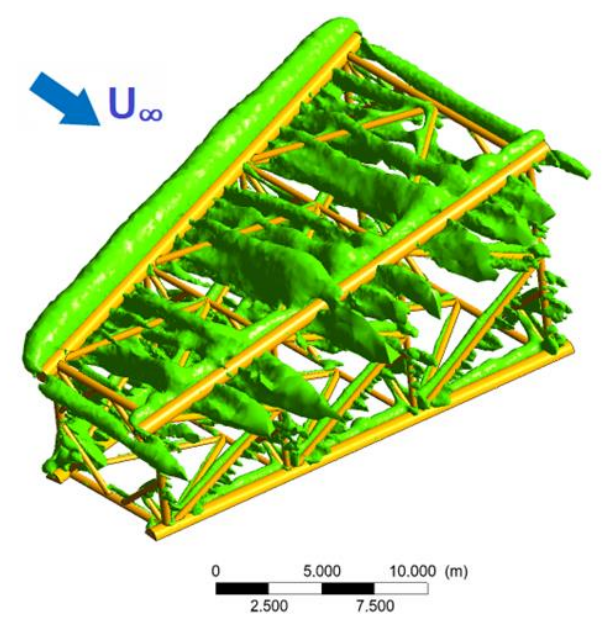

Fig. 15. Upwelling isovolume generated by the flow at $0.45 \mathrm{~m} / \mathrm{s}$ passing through the toppled jacket at $90^{\circ}$ to the prevailing current

Regarding the volume of the back eddy formation, this condition also brings the most favorable result of all, in terms of relative volume. Figure 16 shows the respective isovolume for this case.

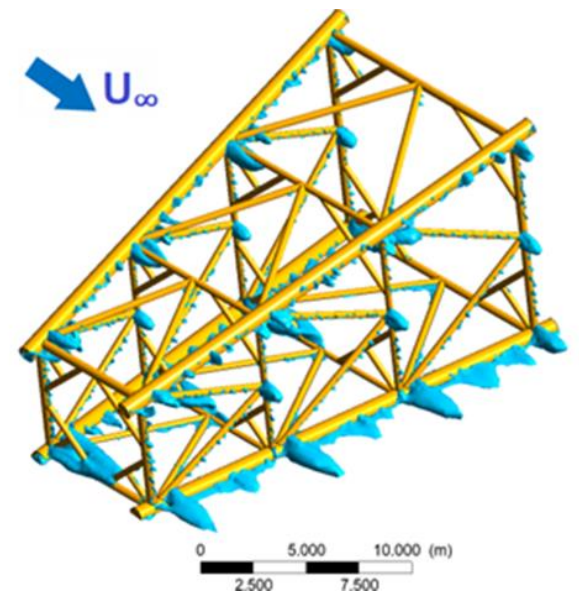

Fig. 16. Back eddy isovolume obtained by the flow at $0.45 \mathrm{~m} / \mathrm{s}$ through the jacket at $90^{\circ}$ to the prevailing current

All other cases are shown to be less relevant in terms of back eddy formation, with toppling in an angle of $135^{\circ}$ to the flow showing the lowest relative volume. The complete comparison of all back eddy relative volumes can be seen in the graph below (Figure 17).

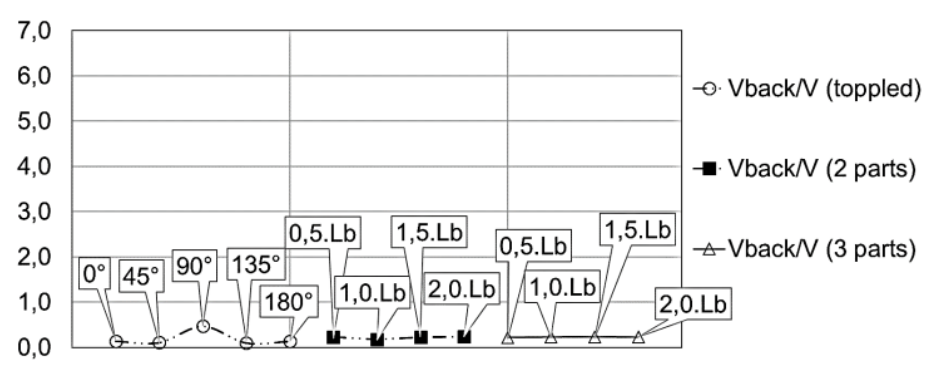

Fig. 17. Variation of $V_{\text {back }} / V$ for each case 
It is observed that the strategy of separating the structure in two parts presents no significant advantages to the others, tending to generate intermediate upwelling and back eddy volumes.

\subsection{Result Discussion}

The disassembly strategy presented by case " $3 . a$ " is advantageous in terms of upwelling generation, which is one of the most important factors for the efficiency of the AR, in terms of life attraction.

Those results suggest that the arrangement of the structures in a great number of parts arranged side by side and increasing the height tends to generate larger upwelling regions. The velocity distribution shown in Figure 18 magnifies the gradual flow disturbance that occurs in this type of arrangement.

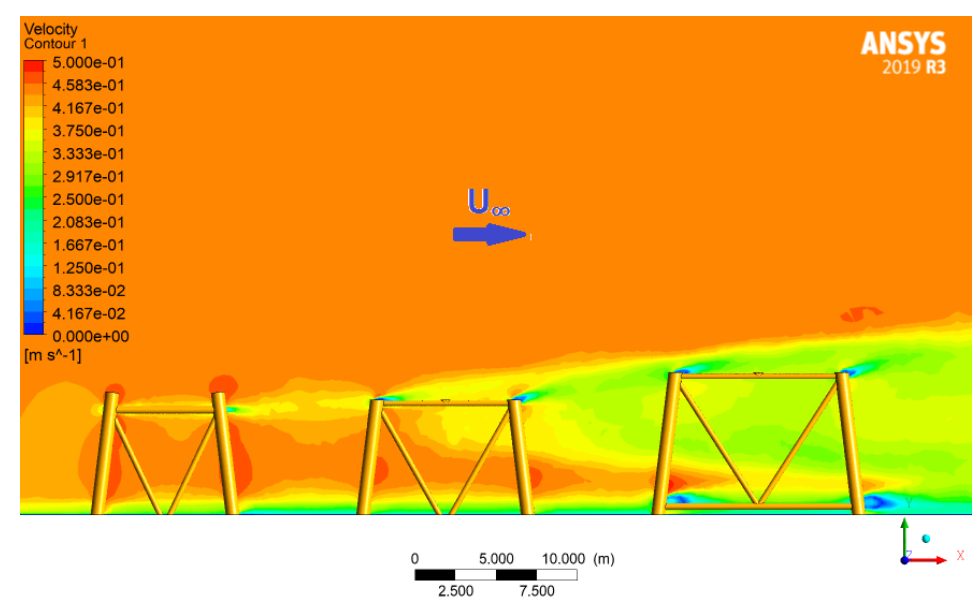

Fig. 18. Velocity distribution generated by the flow at $0.45 \mathrm{~m} / \mathrm{s}$ passing through the structure cut into 3 parts spaced by $0.5 L_{b}$

It is also notable that in the case for the jacket toppled at $180^{\circ}$ to the prevailing current, there is also a gradual increase in the height of the AR. However, the smoothness and continuity of the structure ends up reducing the disturbances induced to the flow, making the effects of back eddy and upwelling less prominent than any other cutting and sinking mode. This can be clearly seen in Figure 19.

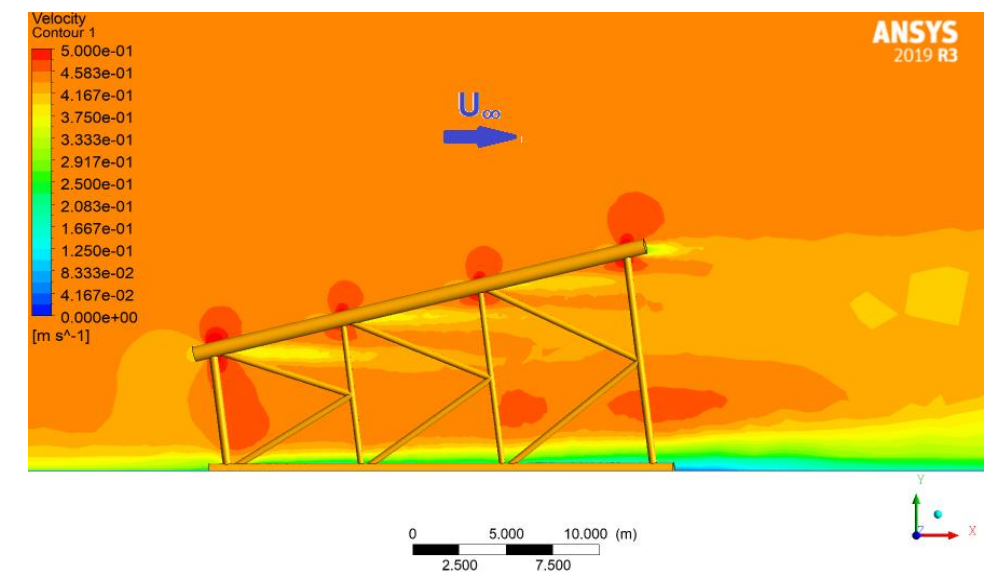

Fig. 19. Velocity distribution generated by the flow at $0.45 \mathrm{~m} / \mathrm{s}$ passing through the structure toppled at $180^{\circ}$ streamwise 
The strategy of cutting the jacket in tree parts has two additional advantages: 1) the maximum height of the AR is smaller, which allows the execution of this project at smaller depths without impacting navigation, 2) also, the base of the structure can be maintained in place, partially preserving the biology that is already aggregated. Sammarco, [4], demonstrated that during the transport and toppling of a jacket, more fragile species tends to reduce significatively. However, it may arise some technical difficulties and cost aggregators since it requires more than one cutting operation.

A suggestion that can be brought from this, considering complexes oilfields, is the association of different jackets that could be brought together and positioned in the streamwise direction, as seen in Figure 20.

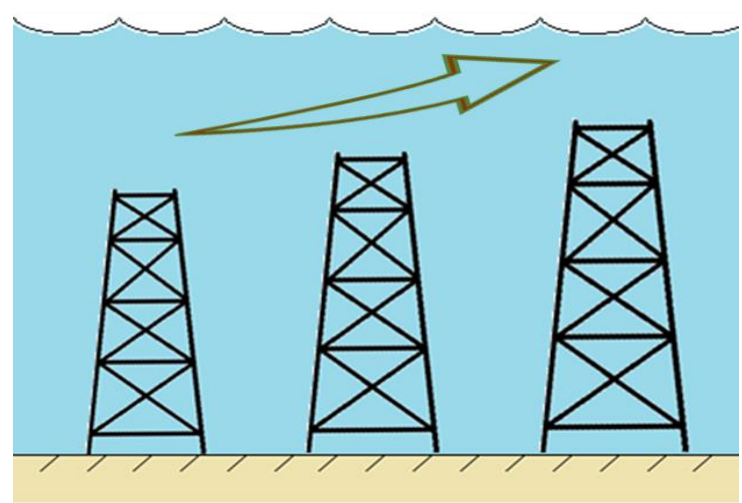

Fig. 20. Hypothetical association of different jackets with similar sizes brought together

In case of unfeasibility of disassembling the jacket in parts or associating with other structures, then tipping it in an angle of $90^{\circ}$ in relation to the prevailing marine current should be strongly considered.

\section{Conclusions}

The use of inoperative jacket type platform structures for sinking and conversion to artificial reefs becomes an attractive alternative for oil and gas exploration companies, since it allows costs reduction for transport and allocation of large structures to land, in addition to favor the recovery of marine biodiversity, also bringing benefits to local fishing communities. And as most fixed type platforms in Brazil are in the Northeast region, where the current velocity conditions are favorable (below $0.5 \mathrm{~m} / \mathrm{s}$ ), then there is a great potential for this alternative in that country.

However, detailed engineering and planning are essential to the success of this type of endeavor. In this context, the study of the hydrodynamic characteristics around decommissioned jacket becomes necessary.

In a comparison between several combinations of cutting and sinking one jacket, the side-by-side arrangement with increasing height streamwise is the one that presents the best results in terms of local upwelling generation, making a good alternative for benthic and sessile species development. Similarly, different jackets can be brought together and positioned in a streamwise direction with increasing height.

Regarding the formation of back eddy regions, it is noted that in the ARs converted from decommissioned jackets, this tends to be a less significant effect. By tipping the jacket at $90^{\circ}$ in relation to the prevailing current, it is observed a higher eddy recirculation volume, making this 
alternative recommended when cutting in three or more sections is unfeasible and/or if the AR is being projected to maximize fish development.

The results of this work are only indicating the path to be followed to determine the most effective way of dismantling and sinking these kinds of structures for an AR conversion.

Future studies can be conducted in transient regimes, seeking to obtain the effects of vortex shedding on the jacket surface in a more detailed way. It is also suggested to evaluate the phenomenon of local drag and hydrodynamic pressure, thus allowing to find and reduce spots that may be unfavorable to the adhesion of larvae and microbiological species. It is highly recommended to carry out studies in current channels for comparison with experimental results.

\section{Acknowledgement}

The authors acknowledge Fluminense Federal University for its educational support and supply of licenses for the ANSYS code. Also, they also express appreciation to the reviewers for all remarks and suggestions. This research was not funded by any grant.

\section{References}

[1] Kaiser, Mark J. "Offshore decommissioning cost estimation in the Gulf of Mexico." Journal of construction engineering and management 132, no. 3 (2006): 249-258. https://doi.org/10.1061/(ASCE)07339364(2006)132:3(249)

[2] Zalmon, Ilana R., Ronaldo Novelli, Marcelo P. Gomes, and Vicente V. Faria. "Experimental results of an artificial reef programme on the Brazilian coast north of Rio de Janeiro." ICES Journal of Marine Science 59, no. suppl (2002): S83S87. https://doi.org/10.1006/jmsc. 2002.1273

[3] Baine, Mark. "Artificial reefs: a review of their design, application, management and performance." Ocean \& Coastal Management 44, no. 3-4 (2001): 241-259. https://doi.org/10.1016/S0964-5691(01)00048-5

[4] Sammarco, P. W., A. Lirette, Y. F. Tung, G. S. Boland, M. Genazzio, and J. Sinclair. "Coral communities on artificial reefs in the Gulf of Mexico: standing vs. toppled oil platforms." ICES Journal of Marine Science 71, no. 2 (2014): 417426. https://doi.org/10.1093/icesjms/fst140

[5] Baynes, Tracy W., and Alina M. Szmant. "Effect of current on the sessile benthic community structure of an artificial reef." Bulletin of Marine Science 44, no. 2 (1989): 545-566.

[6] Lindberg, W. J., William Seaman, and Dorothy Zimmerman. Guidelines and management practices for artificial reef siting, use, construction, and anchoring in southeast Florida. Florida Department of Environmental Protection, 2011.

[7] Ito, Yasushi. "Artificial reef function in fishing grounds off Japan." Artificial Reefs in Fisheries Management (2011): 239-264.

[8] Zheng, Yan-xuan, Zhen-lin Liang, Chang-tao Guan, Xie-fa Song, Jiao Li, Yong Cui, Qiang Li, and You Zhou. "Numerical simulation and experimental study of the effects of disposal space on the flow field around the combined threetube reefs." China Ocean Engineering 29, no. 3 (2015): 445-458. https://doi.org/10.1007/s13344-015-0031-1

[9] Liu, Yan, Chang-Tao Guan, Yun-Peng Zhao, Yong Cui, and Guo-Hai Dong. "Numerical simulation and PIV study of unsteady flow around hollow cube artificial reef with free water surface." Engineering Applications of Computational Fluid Mechanics 6, no. 4 (2012): 527-540. https://doi.org/10.1080/19942060.2012.11015440

[10] Liu, Yan, Yun-peng Zhao, Guo-hai Dong, Chang-tao Guan, Yong Cui, and Tiao-Jian Xu. "A study of the flow field characteristics around star-shaped artificial reefs." Journal of Fluids and Structures 39 (2013): 27-40. https://doi.org/10.1016/i.jfluidstructs.2013.02.018

[11] Rahman, Mohd Asamudin A., Muhammad Nadzrin Nazri, Ahmad Fitriadhy, Mohammad Fadhli Ahmad, Erwan Hafizi Kasiman, Mohd Azlan Musa, Fatin Alias, and Mohd Hairil Mohd. "A Fundamental CFD Investigation of Offshore Structures for Artificial Coral Reef Development." CFD Letters 12, no. 7 (2020): 110-125. https://doi.org/10.37934/cfdl.12.7.110125

[12] Rahman, Mohd Asamudin A., Muhammad Nadzrin Nazri, Fatin Alias, Ahmad Fitriadhy, and Mohd Hairil Mohd. "Computational Fluid Dynamics Analysis of Rigs-to-Reefs (R2R) Jacket Structures." CFD Letters 13, no. 1 (2021): $72-$ 83. https://doi.org/10.37934/cfdl.13.1.7283

[13] Thurman, Harold V. Essentials of Oceanography. 2019.

[14] Kim, Dongha, Jinho Woo, Han-Sam Yoon, and Won-Bae Na. "Wake lengths and structural responses of Korean general artificial reefs." Ocean Engineering 92 (2014): 83-91. https://doi.org/10.1016/j.oceaneng.2014.09.040 
[15] Jiang, Zhaoyang, Zhenlin Liang, Yanli Tang, Liuyi Huang, Dingyong Yu, and Mansong Jiang. "Numerical simulation and experimental study of the hydrodynamics of a modeled reef located within a current." Chinese Journal of Oceanology and Limnology 28, no. 2 (2010): 267-273. https://doi.org/10.1007/s00343-010-9228-6

[16] Yaakob, Omar B., Yasser M. Ahmed, M. Rajali Jalal, A. A. Faizul, Koh K. Koh, and Tarmizi J. Zaid. "Hydrodynamic Design of New Type of Artificial Reefs." In Applied Mechanics and Materials, vol. 819, pp. 406-419. Trans Tech Publications Ltd, 2016. https://doi.org/10.4028/www.scientific.net/AMM.819.406

[17] International Maritime Organization (IMO), Resolution A.672(16), Guidelines and Standards for the Removal of Offshore Installations and Structures on the Continental Shelf and in the Exclusive Economic Zone. 1989.

[18] Versteeg, Henk Kaarle, and Weeratunge Malalasekera. An introduction to computational fluid dynamics: the finite volume method. Pearson education, 2007.

[19] Brazilian Navy PNBOIA Data. 2020.

[20] Sorensen, Robert M. Basic Wave Mechanics: for Coastal and Ocean Engineers. John Wiley \& Sons, 1993.

[21] Veritas, Det Norske. "Free spanning pipelines." Recommended practice DNV-RPF105 (2006): 1-46.

[22] Ali, Arifah, Adi Maimun, and Yasser Mohamed Ahmed. "Analysis of Resistance and Generated Wave around Semi SWATH Hull at Deep and Shallow Water." Journal of Advanced Research in Fluid Mechanics and Thermal Sciences 58, no. 2 (2019): 247-260.

[23] Pope, Stephen B. Turbulent flows, 2001. https://doi.org/10.1017/CB09780511840531

[24] Schlichting, Hermann, and Klaus Gersten. Boundary-layer theory. Springer, 2016. https://doi.org/10.1007/978-3$\underline{662-52919-5}$ 